\section{What is a UDA?}

\section{Michael Watson replies.}

\section{UDAs and DCPs}

Units of Dental Activity (UDAs) are probably the most misunderstood part of the 2006 NHS dental contract. ${ }^{1}$ Their essential purpose is to measure a practice's activity and from that to ensure that the correct amount of patients' charges is collected. The Primary Care Trust (England) or Local Health Board (Wales) set targets to be achieved. If these are missed the contract holder and the practice may be financially penalised. Dental care professionals (DCPs) contribute towards achieving these targets.

\section{What are UDAs?}

UDAs are essentially the value given to a course of treatment (CoT); for example 1 UDA for a CoT including an examination - up to 12 UDAs for a CoT that includes some laboratory work. Since practices are paid according to the number of courses of treatment they complete then a CoT involving some laboratory work is worth 12 times that of a CoT involving only an examination.

The important point to grasp is that under the old NHS contract dentists were paid for every item of treatment they provided: examination, filling, crown or denture. Under the new system they are paid per course of treatment, irrespective of how many items are provided within it. Thus a course of treatment involving one filling (3 UDAs) attracts the same fee as one containing five fillings, a root treatment and an extraction (also 3 UDAs).

This factor is behind much of the resentment against this system.

The number of UDAs that can be claimed is related to treatment bands laid out in the patients' charges regulations. ${ }^{2}$ Despite this they are claimed both for those patients who pay charges and those who are exempt (including children). They are divided into four main headings:

- Band 1 - clinical examination, radiographs, scaling and polishing, preventive dental work, such as oral health advice - 1 UDA

- Band 1 (urgent) - treatment including examination, radiographs, dressings, recementing crowns, up to two extractions, one filling - 1.2 UDAs

- Band 2 - simple treatment, for example fillings, including root canal therapy, extractions, surgical procedures and denture additions - 3 UDAs
- Band 3 - complex treatment, which includes a laboratory element, such as bridgework, crowns, and dentures - 12 UDAs.

UDAs can also be claimed for treatments that do not have a patient's charge, such as issue of prescription (0.75 UDAs), denture repair (1 UDA) and arrest of bleeding (1.2 UDAs).

\section{Who can earn UDAs?}

Only those who hold a GDS (General Dental Services) contract $^{3}$ can earn or claim UDAs. This is usually the practice owner or corporate body, known as the Contractor. Although associates ${ }^{4}$ are often paid per UDA, they are carrying out these treatments on behalf of the practice owner and the UDAs they deliver are credited to the practice total.

In a similar way, if a hygienist or therapist carries out the treatment for a patient, the UDAs they deliver are credited to the practice total, as happens with an associate. The same would apply to an orthodontic therapist or clinical dental technician working to a dentist's prescription. Part of the 12 UDAs for a Band 3 course of treatment, however, is there to pay the laboratory fees. Nowhere, however, does it lay down how what proportion of the money those UDAs earn is intended for laboratories. But dentists receive some of these UDA earnings to pay their technicians.

What this all boils down to is that contract holders, therefore practices earn the UDAs and everyone within the dental team, in the practice or outside it, make their own contributions to earning them. This applies whether they are dentists, who have direct clinical responsibility for the course of treatment, hygienists, therapists, dental nurses or receptionists.

\section{The team - scope of practice}

In team sports all players have their own positions on the field. We do not expect the guy who scores goals at one end to rush back and save them at the other. So it should be in a dental practice. The General Dental Council (GDC) recently brought out a document on the scope of practice ${ }^{5}$ of each dental team member. It is worth a read.

A hygienist can 'plan the delivery of care for patients to improve and maintain their periodontal health'; this will help towards delivering either 1 or 3 UDAs for such care. A dental nurse could develop skills in oral health
'In team sports

all players

have their own

positions on the

field. We do not

expect the guy

who scores goals

at one end to

rush back and

save them at

the other. So it

should be in a dental practice.'

education and oral health promotion, as well as applying fluoride varnish. This will help deliver prevention and earn UDAs.

\section{Steele - the blended contract}

The standard contract introduced in April 2006 is changing with innovative primary care trusts. Starting this year, the Steele report ${ }^{6}$ will be piloted introducing the so-called 'blended contract'. This will involve payments for patients registered by the practice, rewards for quality, but also for activity. The latter means that UDAs will form a lesser part of a future contract, but they will remain. They may be modified, but the activity needs to be recorded and rewarded. UDAs are not going away.

1. The National Health Service (General Dental Services Contracts) Regulations 2005.

2. The National Health Service (Dental Charges) Regulations 2005_Schedules 1-4.

3. Or Personal Dental Services (PDS)

Agreement.

4. Known in the regulations as 'performers'.

5. Scope of Practice - General Dental Council: www.gdc-uk.org.

6. NHS dental services in England: an independent review led by Professor Jimmy Steele. Department of Health, June 2009. 